\title{
Tomás IBÁÑEZ
}

\author{
FACTORES SOCIALES DE LA PERCEPCIÓN \\ HACIA UNA PSICOSOCIOLOGIA DEL SIGNIFICADO
}

\section{LA CONSTRUCCIÓN/INTERPRETACIÓN DE LA REALIDAD}

El tema que intentaré exponer abarca tan solo un reducido aspecto de lo que, a mi entender, constituye la tarea más apremiante y quizás más apasionante de la psicosociología: explicar los procesos (eminentemente sociales puesto que implican pensamiento, comunicación, lenguaje, etc. ...) por los que el ser humano construye/interpreta la realidad. Realidad que no diferenciaré, como es usual, en física y social por entender que toda la realidad que el hombre es capaz de construir/interpretar está densamente investida por factores sociales. Ninguno de los objetos que penetran en el campo sensorial del hombre y, a fortiori, en su campo representacional escapan a los determinismos sociales aun y cuando no se les asignen funciones o valores sociales explícitos ${ }^{1}$.

I La razón es análoga a la que hace que a partir de un código sólo puedan engendrarse mensajes pertenecientes a su propio universo del discurso. 
Creo que la construcción/interpretación de la realidad constituye el objeto a dilucidar prioritariamente por la sencilla razón de que el hombre no reacciona ante la realidad tal y como es si no ante la realidad tal y como él la construye/interpreta. Esta afirmación no necesitaría mayores desarrollos pero la influencia que sigue ejerciendo la corriente positivista-conductista (aun en sus versiones neo positivistas - neo conductistas) nos obliga a mencionar los trabajos de un $\mathrm{Ph}$. Zimbardo y más especialmente sus observaciones dramáticas acerca de los asilos en donde los ancianos se mueren o sobreviven según la interpretación que den a su situación, sus reflexiones acerca de las muertes tipo Vudu y sus investigaciones sobre el control cognoscitivo del dolor (ZIMBardo, 1973). También se podría citar el interesante experimento ${ }^{2}$ de SCHACHTER y SINGER (1962) en el cual se ve cómo una misma droga (Epiphrenina) inyectada a distintos sujetos provoca o no unos estados afectivos particulares según el tipo de interpretación que el sujeto pueda dar a sus sintomas.

$\mathrm{Ni}$ que uecir tiene que el problema planteado por la investigación de la construcción/interpretación de la realidad es enorme ya que abarca toda la problemática de la lectura de la experiencia así como la constitución y el funcionamiento de los procesos semiológicos que engendran los tamices de lectura y plantea en definitiva todo el problema del funcionamiento y función de las ideologías.

\section{RELATIVIDAD Y CATEGORIZACIÓN}

Dentro de este amplísimo campo destacaremos aquí dos importantes procesos de orden inferencial: el proceso comparativo y el proceso de categorización.

Toda interpretación de la experiencia, sobre todo en sus dimensiones evaluativas, implica necesariamente un proceso comparativo. La actividad judicatoria es por definición una actividad de chequeo, de confrontación entre la experiencia a enjuiciar y ciertos criterios o patrones que sirven de marco de referencia. En este sentido se puede afirmar que toda experiencia es relativa en relación al patrón con que se mide.

Todos sabemos, por ejemplo, que un determinado nivel de remuneración que se venía aceptando mal o bien aparece de repente como totalmente inaguantable cuando uno se entera de que por el mismo trabajo y cualificación otros ganan más. De hecho toda la teoría de los grupos de referencia así como los estu-

2 Rechazar el conductismo no significa adentrarse en la fenomenología y rehuir la experimentación. 


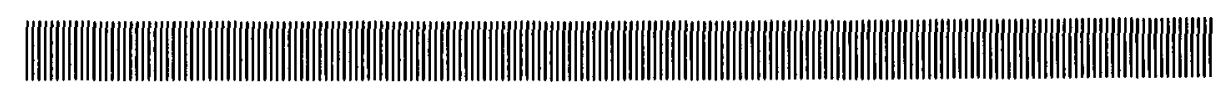

dios en torno a los niveles de espectación y de estima de si mismo ilustran perfectamente el aspecto comparativo que yace en toda experiencia.

Pero la experiencia además de implicar comparación y relatividad también implica categorización. Esta noción nos conecta directamente con los estudios, ya clásicos, del New look in perception, ilustrado por los nombres de Bruner, Goodman, Postman, etc. y con el énfasis que ponía esta corriente teórica en los procesos inferenciales de la percepción: pre-inferencias perceptivas, hipótesis perceptivas, etc. ... El eje teórico del New look puede resumirse en el famoso «ir más allá de la información efectivamente dada» con que Bruner denominó lo que le parecía constituir una de las características básicas del proceso perceptivo, agregando, con evidentes presupuestos funcionalistas, que esta tendencia a sobrepasar la información proporcionada constituía una condición necesaria para la supervivencia del hombre y para su capacidad de reaccionar con la suficiente rapidez ante los acontecimientos producidos en su entorno. A través de su interacción con la realidad el hombre extrae unos elementos de información pero en vez de agotar el tratamiento de toda la información que esta interacción podría proporcionarle y en vez de aplicar nuevas operaciones a la realidad para completar esta información, el individuo se limita a tratar unos fragmentos de información y a extrapolar, a partir de ellos, la naturaleza de los elementos restantes ahorrando, así, tiempo y esfuerzo. El proceso subyacente a este «ir más allá de la información efectivamente dada» es un proceso de categorización que puede definirse, siguiendo los términos de Tajfel, como el conjunto de procesos psicológicos que tienden a ordenar el entorno en términos de categorías, es decir, de conjuntos de objetos, personas, acontecimientos (o bien algunos de sus atributos) en tanto en cuanto son semejantes o equivalentes entre sí respecto a la actuación, las intenciones o las actitudes del individuo.

El proceso de categorización implica un doble aspecto inductivo y deductivo. Inductivo cuando a partir de ciertas características de un objeto se le asigna una determinada categoría de objetos. Deductivo cuando en base a esta asignación se atribuyen al objeto las características y propiedades de su clase de asignación. Ciertas frases pronunciadas por una persona nos inducirán a catalogarla como fascista, por ejemplo, sin que procedamos a mayores averiguaciones y además prestaremos a esta persona rasgos típicos de nuestro estereotipo del fascista sin proceder aquí tampoco a mayores averiguaciones. El modelo probabilístico en que se funda este proceso perceptivo es evidente así como son evidentes los efectos sociales que resultan de la categorización. Además de la tendencia reificadora consustancial con el desarrollo correcto del proceso de categorización podemos intuir las consecuencias de los disfuncionamientos que pueden darse en las distintas fases del proceso. En la fase inductiva ocurre que se atribuye erró- 
neamente un objeto a una clase de pertenencia y las investigaciones de Bruner y Postman han demostrado la extraordinaria resistencia que opone una categorización errónea a la redefinición del objeto y a la reestructuración perceptiva del mismo. Siempre en la fase inductiva ocurren procesos de super-inclusión y super-exclusión particularmente claros en las investigaciones de ALLPORT (1946), acerca de los prejuicios étnicos. En cuanto a la fase deductiva todos sabemos las consecuencias de los estereotipos sobre todo cuando las características del estereotipo son de orden cultural o ideológico. Los que llevan pelo largo, los negros, las mujeres, etc. saben de estas consecuencias ${ }^{3}$.

\section{EL PROBLEMA DE LOS VALORES EN LA PERCEPCIÓN}

Volviendo a la corriente del New Look, es un hecho de todos conocido el que muchas de las investigaciones que se realizaron bajo su influencia teórica tuvieron como objeto dilucidar las incidencias de los «valores" sobre los procesos perceptivos. En un experimento que hizo época BRUNER y GOODMAN (1947) estudiaron las diferencias perceptivas que se producen respecto al tamano de los objetos cuando éstos difieren entre sí «únicamente» en cuanto a su valor para el sujeto. Concretamente estos autores pedían a una población de nifos de diez ańos que estimasen las dimensiones de una serie de monedas (de $1 \$$ a 50 cts.) ajustando un haz luminoso graduable que reproduciese sus dimensiones y que estimasen por otra parte y con el mismo procedimiento unos discos de cartón de idénticas dimensiones que las monedas. Bruner y Goodman formulaban la hipótesis de que se produciría una sobre-estimación sistemática de las monedas y solamente de las monedas y que esta sobre-estimación sería tanto más importante cuanto mayor valor tuviesen las monedas. Bruner y Goodman observaron efectivamente una tendencia a la sobre-estimación de las monedas y demostraron incluso que esta sobre-estimación era tanto más acentuada cuanto más "pobres" eran los sujetos.

Sin embargo ulteriores réplicas de este experimento en que se utilizaban también discos de metal junto con los discos de cartón no permitieron comprobar la hipótesis de Bruner y Goodman. Otros experimentos en los que se utilizaron distintos materiales (símbolos, fichas canjeables por caramelos, etc. ...) arroja-

3 Recordemos la definición que dan SECORD y BACKMAN (1964) de la actividad de estereotipificación: acción de asignar atributos a una persona basándose únicamente en la clase o categoría a la cual pertenece. 
\|\|$\|$

ron resultados contradictorios que confirmaban o invalidaban la hipótesis según los casos. Finalmente Bruner abandonó su hipótesis ya que el valor de los objetos parecía conducir a una sobre-estimación en ciertos casos y no en otros.

\section{TAJFEL Y LAS VARIACIONES SOBRE-IMPUESTAS (superimposed)}

Analizando los resultados contradictorios proporcionados por el conjunto de experimentos en torno a la hipótesis Bruner-Goodman y reflexionando acerca de las diferencias y similitudes entre los procesos perceptivos estudiados por la psicofísica y los procesos perceptivos estudiados en el área social, Tajfel desembocó en la formulación de una teoría que permite a la vez:

a) Explicar la aparente contradicción de los datos recogidos.

b) Reducir el problema del valor en la percepción a un simple caso particular de un proceso mucho más general.

El estudio psicofísico de la percepción se desarrolla según un paradigma en el cual los objetos sólo deben diferenciarse entre sí en base a una única dimensión de variabilidad que denominaremos su dimensión focal (peso, talla, color, etc. ...). Esta es una condición necesaria para la validez de las investigaciones y ahí está para recordárnoslo la triste experiencia de Fechner que consideró como desperdiciado todo un año de investigaciones cuando se dio cuenta de que habia utilizado unos objetos que no sólo variaban entre sí respecto a la dimensión estudiada (el peso) sino que se diferenciaban también respecto a la localización de su centro de gravedad.

Sin embargo, el individuo en su vida cotidiana, no suele verse confrontado con objetos que varían en una sola dimensión sino que se encuentra frente a unas series de objetos que se diferencian entre sí en una multiplicidad de dimensiones a la vez. En un terreno tan familiar para nosotros como es el de las actitudes, todos tenemos consciencia, por ejemplo, de que dos enunciados actitudinales insertos en una escala, no sólo se diferencian en cuanto al grado de favorabilidad que implica su aceptación/rechazo por los sujetos sino que también se diferencian en relación al número de palabras que tienen, a la estructura sintáctica, a la frecuencia de ocurrencia de las palabras en el vocabulario, etc., etc. ..., iinciden estas diferencias no controladas sobre lo que pretendemos medir? Dejo la pregunta abierta ya que este ejemplo no tenía otro objeto que ilustrar la afirmación de que los objetos suelen diferenciarse pluridimensionalmente aunque por determinadas razones sólo se tome en consideración una de estas dimensiones. 
La teoría elaborada por Tajfel se centra justamente sobre este aspecto de las variaciones múltiples aunque sólo rinda cuenta de las variaciones bidimensionales, Es decir, que intenta explicar lo que ocurre cuando los objetos de una serie determinạda varian los unos en relación con los otros en dos dimensiones simultáneamente. Esta co-variación de las diferencias entre los objetos puede darse en base a una relación constante y. sistemática o bien en base a una relación ella misma variable y aparentemente aleatoria. Por ejemplo, el tamaño y el precio de los turismos suelen variar é̉ correlación, es decir, que una información acerca de la diferencia de tamaño entre dos coches constituye un predictor aceptable del sentido en que varia la diferencia de precio entre esos dos coches, mientras que otra dimensión sobre la cual también se diferencian los coches como es el color no guarda una relación fija, o no está en correlación, con el tamano de éstos. La teoría de Tajfel se cine a los casos en que las variaciones se presentan en correlación. La pr ugunta concreta a-la que intenta responder la teoria es la de saber: ¿qué ocurre respecto a los procesos perceptivos cuando los objetos de una serie se diferencian antre sí por dos aspectos simultáneos y correlacionados? Por ejemplo, ¿qué ocurre cuando unos objetos se diferencian a la vez por su tamafio y su valor (monedas), y las diferencias de tamaño y valor presentan una relación constante (a mayor valor mayor tamáno)?, o bien, ¿qué ocurre cuando unos objetos presentan entre sí diferentias correlacionadas respecto a su color y respecto a sus dimensiones, etc.?

\section{LA TEORÍA DE LA ACENTUACIÓN}

La hipótesis de TAJFEL (1959) es que cuando los estímulos de una serie se diferencian en dos o más dimensiones de forma simultánea y correlacionada, entonces se incrementa la discriminabilidad entre estos objetos. Recogiendo la distinción entre dimensión focal lo dimensión de evaluación, o dimensión sobre la cual se centra el sujetol y dimensión periférica, se puede decir que las variaciones que se producen en las dimensiones periféricas constituyen diferencias superimpuestas a las variaciones que se producen en la dimensión. focal.

Tajfel predice que en estos casos se observará una acentuación de las diferencias percibidas entre los objetos en la dimensión focal. Como consecuencia, los estímulos situados en el polo inferior de la serie serán infra-valorados y los estímulos situados en el polo opuesto serán sobre-valorados.

Imaginemos un experimento en el cual se presentan dos series de líneás de distintas longitudes. En la serie I las líneas sólo varían respecto a la longitud, en 


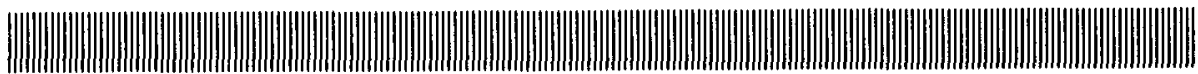

la serie II las líneas idénticas a las de la serie I son además tanto más oscuras cuanto más largas. Según Tajfel las diferencias de longitud se percibirán más acentuadas en la serie Il que en la serie I, y si comparamos las dos series, observaremos que las líneas más cortas de la serie Il serán infra-valoradas con respecto a las líneas correspondientes de la serie I a la vez que las líneas más largas de la serie II serán super-valoradas. Sin embargo, una tercera serie de líneas III en la cual el color más o menos oscuro de las líneas no guarde relación con su longitud no producirá ningún efecto de acentuación.

Esto nos explica los resultados contradictorios obtenidos en la serie de experimentos suscitados por las hipótesis de Bruner ya que solamente puede producirse una sobre-estimación de las dimensiones de los objetos que encierran un valor para los sujetos si existe una correlación entre las variaciones de dimensión y las diferencias de valor.

Es decir, que la hipótesis Bruner-Goodman según la cual los objetos valorados serán sobre-estimados con relación a objetos «neutros» de idénticas dimensiones es efectivamente correcta pero solamente cuando dimensión y valor varían de forma correlacionada. Todos los experimentos que se apartaban de esta condición arrojaban naturalmente resultados que no confirmaban la hipótesis. Por otra parte el problema del "valor» tal y como lo abordaron Bruner-Goodman se ve reducido a un simple caso particular ya que la sobre-estimación se produce según Tajfel siempre que se presenta una variación "sobre-impuesta" a las variaciones situadas en la dimensión focal, sea cual fuere la naturaleza de esta variación sobre-impuesta.

\section{LAS CATEGORÍAS Y LA TEORÍA DE LA ACENTUACIÓN}

Según Tajfel su teoría es también válida en los casos en que las variaciones en la dimensión periférica no son variaciones continuas, es decir, cuando el número de variaciones en la dimensión periférica no es idéntico al número de variaciones sobre la dimensión focal. En estos casos los estimulos se encuadran en distintas clases de pertenencia definidas por las variaciones en la dimensión periférica. En el experimento acerca de las series de lineas podemos imaginar que en la serie II en vez de una graduación del color en función de la longitud se coloree en rojo la mitad de las líneas (las líneas más largas por ejemplo) y en negro las otras líneas. En este caso Tajfel habla de categoría sobre-impuesta y predice que se observará una acentuación de las diferencias percibidas entre los dos grupos de líneas. Si hay ocho líneas la diferencia entre la cuarta y la quinta (límites de 
las clases) será sobre-estimada. De forma general la teoría de Tajfel predice que se manifiesta una acentuación de las diferencias inter-clases y que siempre que dos o varios estímulos pertenezcan a clases distintas se producirá una exageración de las diferencias percibidas entre estos estímulos.

Ulteriormente TAJFEL y WILKES (1963) han especificado más esta teoria de forma que abarque también la evaluación intra-clase. La pertenencia a distintas clases no sólo provoca una acentuación de las diferencias inter-clases sino que también provoca una acentuación de las similitudes intra-clase. Es decir, que se produce una minimización de las diferencias percibidas entre los estímulos que pertenecen a una misma clase.

\section{LOS EFECTOS DE LA MERA CATEGORIZACIÓN}

Procediendo al estudio de la categorización Tajfel ha intentado averiguar cuáles son los efectos que se desprenden del simple hecho de poder categorizar.

En un experimento (TAJFEL y otros, 1971) se pidió a los sujetos que expresaran sus preferencias acerca de dos cuadros atribuidos el uno a Klee y el otro a Kandinsky. Tras la elección se proporcionaba a los sujetos unas matrices de recompensas que les permitía decidir la forma que tomaría la distribución de recompensas monetarias entre los participantes al experimento. Cada sujeto debía conceder las recompensas a distintas parejas de participantes. Estas parejas estaban constituidas por dos participantes pertenecientes a una misma categoría, es decir, que ambos habian preferido Klee o que ambos habian preferido Kandinsky, con lo cual el sujeto debía repartir las recompensas entre dos individuos pertenecientes a su propia categoria o a la categoría adversa. Las parejas podían también ser heterogéneas, con lo cual el sujeto debía repartir las recompensas entre un miembro de su propia categoría y un miembro de la otra categoría. La categoría de pertenencia era êl único dato de identificación de los participantes que se proporcionaba a los sujetos. Las matrices de recompensa estaban disefiadas de forma que las remuneraciones de los dos individuos de cada pareja fueran dependientes la una de la otra (una única decisión por matriz para cada pareja).

Ejemplos de matrices":
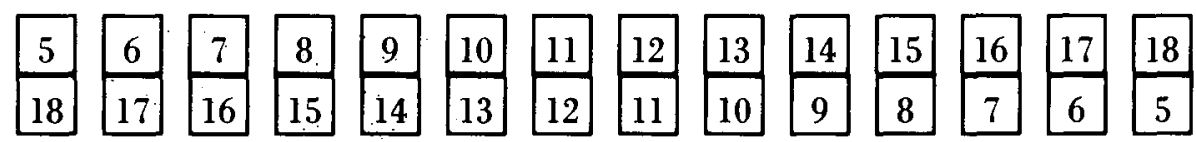

\footnotetext{
4 Los números representan en décimas de penny las retribuciones entre las que debe elegir el sujeto.
} 


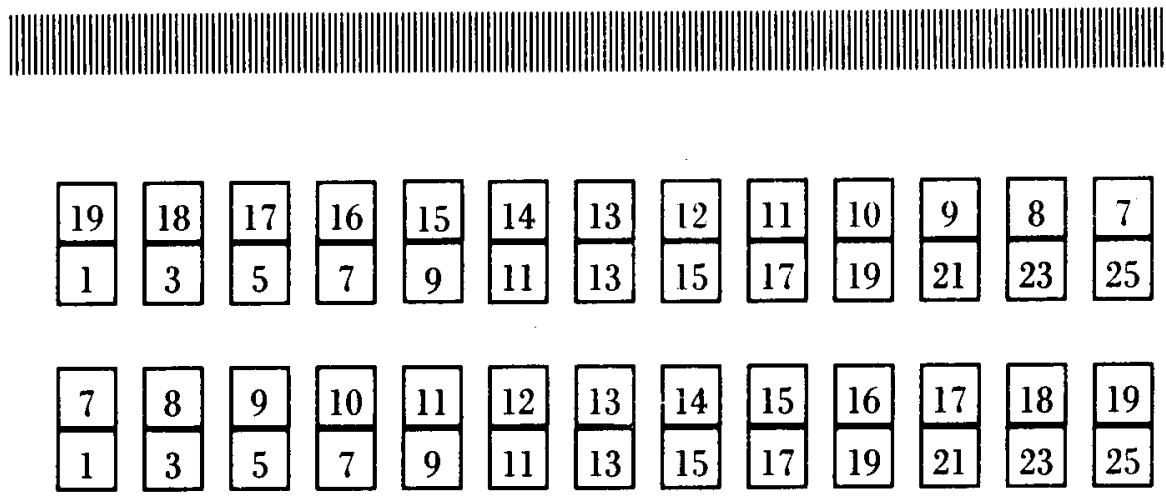

Cuando la pareja a recompensar era heterogénea se precisaba al sujeto cuáles de las casillas de arriba o de las de abajo corresponderían al participante perteneciente a su misma categoría. Está claro que la estructura de matrices permite desarrollar distintas estrategias de recompensa:

Se puede adoptar una estrategia que maximice las ganancias de todos los participantes sean cuales fueran sus preferencias por Klee o por Kandinsky.

Se puede adoptar una estrategia que maximice las ganancias de los miembros de su propia categoría o de la otra categoría.

Se puede adoptar una estrategia que maximice las diferencias de ganancia a favor de los miembros de su propia categoría o de la otra categoría.

De hecho se observa que los sujetos favorecen sistemáticamente los miembros de su propia categoría en vez de adoptar un comportamiento de distribución equitativo de las recompensas o de maximización de las ganancias globales. Incluso los sujetos prefieren reducir en valor absoluto las ganancias de los miembros de su propia categoría con tal de que ganen más que los miembros de la otra categoría (matriz C).

El simple hecho de categorizar a los demás entre "semejantes" a uno mismo (aunque sea sobre aspectos de importancia muy relativa) y «distintos" a uno mismo basta para engendrar comportamientos discriminatorios acerca de los «demás».

\section{CONSECUENCIAS SOCIALES DE LA CATEGORIZACIÓN}

Dejando de lado tanto los aspectos referentes a las sobre-inclusiones y sobre-exclusiones que dependen del valor social atribuido a las categorías, así como los aspectos discriminatorios inducidos por la mera categorización (que hemos discutido en el párrafo anterior) y dejando de lado, también, las consecuencias 
de las categorizaciones erróneas (resistencia a la recepción correcta de la información) quedan sin embargo muchos fenómenos sociales que pueden conectarse con la teoría de la acentuación y con el mecanismo de la categorización... A título indicativo, me parece que se pueden señalar, entre otros, los siguientes:

La inclusión de una persona o de un objeto dentro de una "categoria» sobre la base de un atributo particular propio del elemento categorizado y de la categoría que lo captura promueve una tendencia a atribuir a esa persona u objeto todos los atributos que definen dicha categoría y a actuar con esa persona u objeto como si tuviese efectivamente esos atributos aunque no se tenga información al respecto.

Se tenderá a distorsionar la percepción de las características propias de una persona u objeto de manera que no aparezcan contradicciones entre esas características y las características subjetivamente constitutivas de la categoría de pertenencia.

Dos per 'nnas u objetos que sean percibidas como diferentes en relación a un atributo que permita incluirlas en categorías distintas tenderán a ser percibidas como diferentes, en relación a los restantes atributos que diferencian a las dos categorías de inclusión.

Se tenderá a minimizar las diferencias existentes entre los miembros de un mismo grupo (ya sea étnico, político, etc. ...) en relación a todos aquellos atributos que caracterizan subjetivamente a este grupo (acentuación de la similitud intra-clasel.

Recíprocamente se tenderá a acentuar las diferencias existentes entre los miembros de grupos diferentes.

La pertenencia de una persona a una determinada categoría (social, étnica, etc. ...) influirá más sobre la impresión que nos formemos de ella si la encontramos en compañía de otras personas pertenecientes a otras categorias que si la encontramos sola.

La impresión que nos causa una persona estará tanto menos influenciada por su pertenencia a una categoría social cuanto mejor conozcamos a distintos miembros de esa categoría. (Porque la diversidad que necesariamente habremos hallado entre esos miembros resta fuerza a los efectos de la categorización.)

\section{A MOD́ DE CONCLUSIÓN}

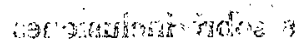

Como deccia al principio, los procesos de categorización no dejan de constituir un fragmento muy reducido del amplio campo que se ofrece a la investiga- 


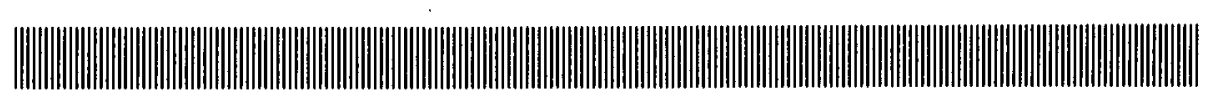

ción de los procesos perceptivos y, sin embargo, el estudio de la categorización permite abordar fenómenos socialmente significativos de las conductas humanas.

A modo de conclusión, me gustaría subrayar con especial énfasis y con ánimo proselitista la pertinencia social que pueden tener las investigaciones que se adentren en el difícil problema de dilucidar los procesos perceptivos. Pero entendiendo percepción en el sentido más amplio de la palabra, sentido que quedaría sin duda mejor explicitado si se substituyese la denominación tradicional de este campo de investigación por la de procesos interpretativos de la realidad, substitución que indica un desplazamiento del enfoque teórico a la vez que implica una opción epistemológica.

\section{BIBLIOGRAFÍA}

Allport, G.W. y Kramer. B., 1946, «Some roots of Prejudice», J. of Psychology, núm., 22, pp. 9.39.

BrunER, J.S. y Goodman C.C., 1947, aValue and need as organizing factor in perception», J. of Social Psychology, núm. 27, pp. 203-208.

Eiser, J.R. y StoEbE, W., 1972, "Categorization and social judgment w, Academic Press. European monographs in social psychology, London, I La influencia de este libro sobre el contenido de mi articulo ha sido considerable).

Secord, P. y Backman, C., 1964, Social Psychology, McGraw-Hill, New York.

TAJFEL, H. y WILKES. A.L., 1963, «Quantitative judgment in social perception», British $J$. of Psychology, núm. 50, pp. 16-29.

Tajfel, H. , Flament, C. y otros, 1971, aSocial categorization and intergroup behavior», European J. of Sacial Psychology, núm. 1, pp. 149-178.

TAJfEl, H. y WILKES, A.L., 1963, "Classification and quantitative judgement», British J. of Phsycology, núm. 54, pp. 101-114.

Zimbardo, Ph., 1969, The cognitive control of motivation, Glenview, Illinois, Scott \& Faresman Co.

- 1972, a La psychologie sociale: une situation, une intrigue et un scenario en qûete de realité», en Moscovici, S. (Ed.), Introduction a la psychologie sociale, Paris, Mouton, Vol. I, pp. 82-102.

Zimbardo, Ph., Cohen, A.R. y otros, 1966, «Control of pain motivation by cognitive Dissonance», Science, núm. 151, pp. 217-219. 